\title{
Erfolgreicher Launch der GTH Mediathek im Juni
}

\author{
Das Wissensportal der Hämostaseologie
}

In diesem Jahr finden viele Fortbildungsveranstaltungen und Kongresse nicht wie geplant statt, so auch unsere GTH Highlights 2020 in Hannover.

Als Fachgesellschaft haben wir uns entschieden, das aktuelle Wissen aus dem Bereich Hämostaseologie als CME-zertifizierte Film- und Audiobeiträge in einer schicken Mediathek zur Verfügung zu stellen.

Wir freuen uns, Ihnen das neueste Angebot und einen vierten Baustein unse-

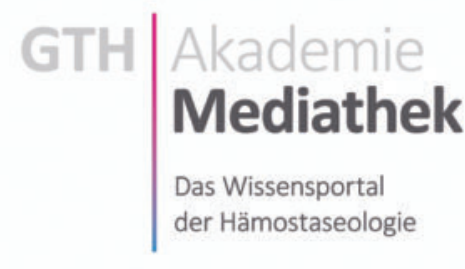
rer stetig wachsenden GTH Akademie vorstellen zu dürfen:

Die GTH Akademie - Mediathek: Das Wissensportal der Hämostaseologie

\section{Wissen up to date}

In der GTH Akademie Mediathek finden Sie seit Juni interessante Vorträge unserer ReferentInnen zu aktuellen Themen der Hämostaseologie wie z.B. Hämostasestörungen bei Covid-19, erworbene Hämophilie, Antikoagulation oder Heparininduzierte Thrombozytopenie. Zu jedem Thema gibt es rund 45 Minuten Film- und Audiomaterial sowie jeweils eine Q\&A-Live-Session mit den Vortragenden. Nach beantworteter Lernerfolgskontrolle erhalten Sie ein Zertifikat und 2 CME Punkte pro Kurs.
Nach der Registrierung auf gth-mediathek.org behält Ihr persönlicher Login bis zum Mai 2021 seine Gültigkeit. Bis dahin stellen wir Ihnen fortlaufend rund 15 aktuelle Kurse mit je drei Lektionen zur Verfügung und informieren Sie per E-Mail über neue Inhalte. Die Schutzgebühr beträgt einmalig 25,00 €, ab Juni 2020 bis Mai 2021 können Sie alle Beiträge jederzeit nutzen.

Weitere Infos auf der Webseite: www.gth-mediathek

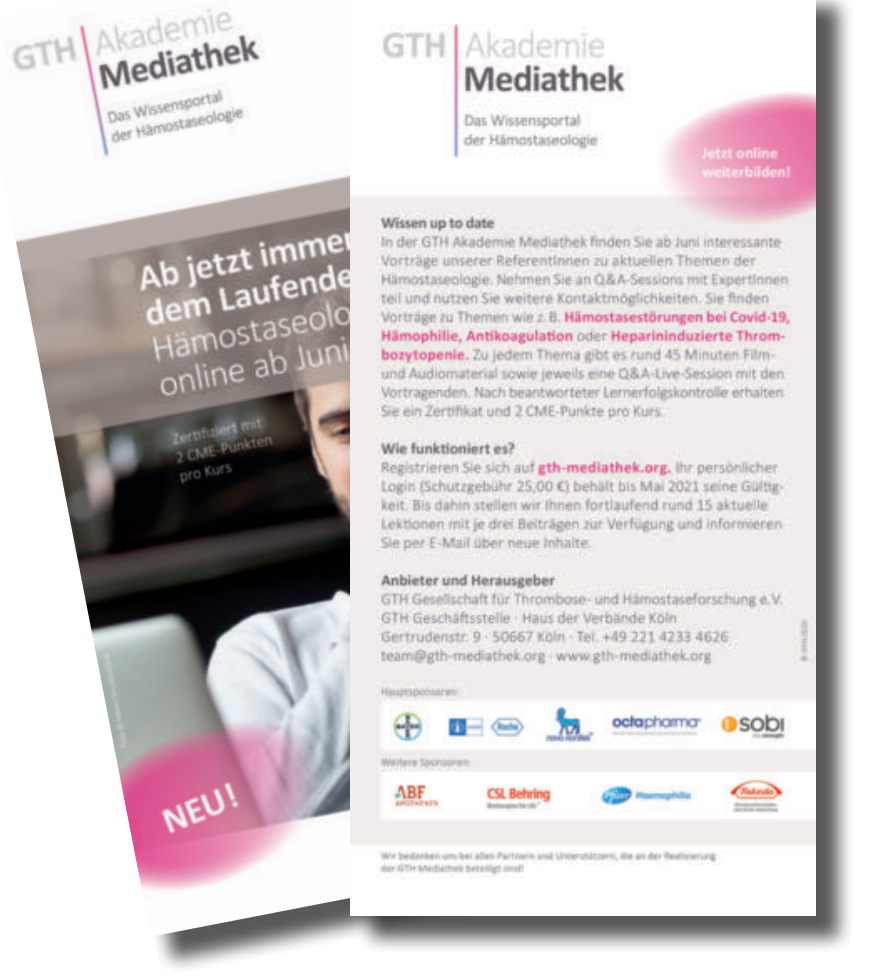

\section{Die Mediathek startet ab Juni mit diesen Beiträgen}

Hämostasestörungen

bei Covid-19

Prof. Dr. Florian Langer

Universitätsklinikum

Hamburg-Eppendorf,

Zentrum für Onkologie,

II. Med. Klinik und Poliklinik

\section{Erworbene Hämophilie}

Prof. Dr. Andreas Tiede

Medizinische Hochschule

Hannover

Klinik für Hämatologie, Hämostaseologie, Onkologie und Stammzelltransplantation

\section{Antikoagulation}

\section{bei Kindern}

Prof. Dr. Christoph Male Medizinische Universität Wien, Univ.-Klinik für Kinder- und Jugendheilkunde

\section{Antiphospholipid- \\ Antikörpersyndrom}

Prof. Dr. Ingrid Pabinger

Medizinische Universität Wien, Klinische Abteilung für Hämatologie und Hämostaseologie 


\section{Early Career Research Grants of the Society of Thrombosis and Haemostasis}

Ab 2021 vergibt die GTH jährlich, befristet auf drei Jahre, drei Early Career Research Grants in Höhe von bis zu 100.000,00€ an Nachwuchsforscher.

Voraussetzungen für Bewerbungen sind ein inhaltlicher Fokus in der Bereichen Hämostase, Thrombose oder vaskuläre Biologie sowie experimentelle, translationale und klinische Themen.

Die Altersgrenze liegt bei 40 Jahren, wobei Kindererziehungszeiten natürlich berücksichtigt werden. Der Arbeitsmittelpunkt sollte in den GTH-Ländern liegen (Deutschland, Österreich, Schweiz) und gern gesehen werden "collaborative" Projekte, z.B. Kooperationen zwischen GTH-Ländern oder Kooperationsprojekte zwischen Forschungsgruppen.

Die Preisverleihung findet im Rahmen des Jahreskongresses der GTH statt.

Bewerbungsschluss für die Grants 2021 ist der 30. November 2020 und in Kürze stehen Antragsformulare sowie detail- lierte Informationen zur Ausschreibung der Preise auf der Webseite der GTH bereit: www.gth-online.org

Weitere Informationen erhalten Sie in der GTH Geschäftsstelle.

\section{GTH $\mid$ Akademie Fortbildungsveranstaltungen der Gesellschaft für Thrombose- und Hämostaseforschung e.V.}

\section{GTH Laborkurs}

\section{September, Leipzig}

für 2020 abgesagt

Der 13. Laborkurs unter Leitung von Ute Scholz in Kooperation mit dem BFSH kann in diesem Jahr leider nicht stattfinden. Der Termin für den Laborkurs in 2021 steht bereits fest: 09.-11.09.2021 Weitere Informationen: www.gth-laborkurs.org

\section{Fortbildung für}

Hämostaseologie-

\section{AssistentInnen}

\section{Juni, Wilsede} verschoben

Auch der Basiskurs Blutungsneigung kann nicht wie geplant stattfinden und wird möglicherweise im November in Hamburg im Rahmen des Hämophilie Symposions angeboten, weitere Entscheidungen dazu fallen Anfang September.

\section{GTH Intensivkurs}

\section{9.-13. November, Gießen}

Die Planung des 25. GTH Intensivkurses läuft aktuell ohne Änderungen. Anmeldungen sind inzwischen ausschließlich online über die Webseite möglich: www.gth-intensivkurs.org.

\section{Impressum}

Verantwortlich für den Inhalt der GTH News:

GTH

Gesellschaft für Thromboseund Hämostaseforschung e.V.
GTH Geschäftsstelle

Haus der Verbände

Gertrudenstr. 9

50667 Köln
Tel. 0221-42334626

mail@gth-online.org

www.gth-online.org 